\title{
TRATAMIENTO FISICOQUÍMICO DE LOS EFLUENTES DEL PROCESO DE LAVADO DE LANA EN UNA INDUSTRIA TEXTIL DE AREQUIPA
}

\author{
María Ofelia Guillén Zevallos*1, Virginia Pérez Murilloa, Tania Quispe Carrizales ${ }^{\mathrm{a}}$, \\ María Elena Talavera Núñez ${ }^{\mathrm{a}}$, Flora Elsa Huamán Paredes ${ }^{\mathrm{b}}$
}

\begin{abstract}
RESUMEN
El objetivo del presente estudio fue evaluar el tratamiento fisicoquímico de los efluentes de lavado de lana provenientes de una industria textil de la ciudad de Arequipa con la finalidad de cumplir con los Valores Máximos Admisibles (VMA), utilizando como coagulante tricloruro férrico al 40 \% y floculante polímero MT-FLOC 4299 al 0,1 \%. Se caracterizó física y químicamente el efluente al inicio y al final del tratamiento, midiendo $\mathrm{pH}$, temperatura, demanda bioquímica de oxígeno (DBO5), demanda química de oxígeno (DQO), sólidos totales suspendidos (TSS), aceites y grasas, sulfatos, sulfuros, nitrógeno amoniacal, cianuro total, cromo hexavalente y turbidez. Para determinar las dosis óptimas de coagulante y floculante se realizaron tres tratamientos utilizando un equipo de jarras (Jar-test) a 50, 200 y $40 \mathrm{rpm}$. El tiempo de sedimentación fue de 10 minutos. Los rangos de turbidez inicial oscilaron entre 200 y 800 NTU obteniéndose una remoción del $89 \%$ en relación a la turbidez promedio de 526,96 NTU. La mejor dosis aplicada al efluente fue $200 \mathrm{mg} / \mathrm{L}$ de tricloruro férrico y $0,8 \mathrm{mg} / \mathrm{L}$ del floculante polímero catiónico MT-FLOC 4299. Los parámetros de remoción de contaminantes fueron: $84,97 \%$ aceites y grasas; $82,55 \%$ demanda bioquímica de oxígeno; 79,36 \% demanda química de oxígeno; 99,60\% sólidos totales suspendidos; $96,67 \%$ sólidos sedimentables y 75,65 \% cianuro total, cumpliendo así con la Normativa Peruana, por lo que es factible aplicar el tratamiento a mayor escala.
\end{abstract}

Palabras clave: Coagulación, floculación, agua residual, textil, lavado de lana.

\footnotetext{
a Facultad de Ciencias Naturales y Formales, Departamento Académico de Química. Universidad Nacional de San Agustín de Arequipa, Santa Catalina N¹17 casilla 23, Arequipa - Perú. *mguillenz@unsa.edu.peb Centro de Investigación en Química, Toxicología y Biotecnología Ambiental, Departamento Académico de Química, Facultad de Ciencias, Universidad Nacional Agraria La Molina, Lima - Perú.
} 


\title{
PHYSICOCHEMICAL TREATMENT OF THE EFFLUENTS OF THE WOOL WASHING PROCESS IN A TEXTILE INDUSTRY OR AREQUIPA
}

\begin{abstract}
The objective of this study was to evaluate the physicochemical treatment of wool washing effluents from a textile industry in the city of Arequipa in order to comply with the Maximum Admissible Values (VMA), using 40\% ferric trichloride as coagulant and 0,1\% MT-FLOC 4299 polymer flocculant. The effluent was physically and chemically characterized at the beginning and at the end of the treatment, measuring $\mathrm{pH}$, temperature, Biochemical Oxygen Demand (BOD), Chemical Oxygen Demand (COD), total suspended solids (TSS), oils and fats, sulfates, ilosulphides, ammoniacal nitrogen, total cyanide, hexavalent chromium and turbidity. To determine the optimal doses of coagulant and flocculent, three treatments were done using a jug set at 50, 200 and $40 \mathrm{rpm}$. The sedimentation time of the floc was 10 minutes. The initial turbidity ranges oscillated between 200 and 800 NTU, obtaining a removal of 89 $\%$ in relation to the average turbidity of 526.96 NTU. The best dose applied to the effluent was $200 \mathrm{mg} / \mathrm{L}$ of ferric trichloride and $0,8 \mathrm{mg} / \mathrm{L}$ of the cationic polymer flocculent MTFLOC 4299. The contaminant removal parameters were: $84,97 \%$ oils and fats; $82,55 \%$ Biochemical Oxygen Demand; 79,36 \% Chemical Oxygen Demand; 99,60 \% suspended total solids; $96,67 \%$ settleable solids and $75,65 \%$ total cyanide, thus complying with the Peruvian Regulations, so it is feasible to apply the treatment on a larger scale.
\end{abstract}

Key words: Coagulation, flocculation, wastewater, textile, wool washing.

\section{INTRODUCCIÓN}

Muchas son las industrias que generan aguas residuales que afectan la calidad del agua. Una de ellas es la industria textil de la ciudad de Arequipa, que en su proceso de lavado genera aguas residuales que contienen alto contenido de materia orgánica y sales inorgánicas, así como elevada DBO5 (demanda bioquímica de oxígeno), DQO (demanda química de oxígeno), grasas, sulfuros, y otros que dañan el sistema de alcantarillado, canales de regadío, generando riesgo potencial a la salud de las personas si se vierten sin tratamiento alguno ${ }^{1}$.

El efluente textil es uno de los tipos de aguas residuales más peligrosas para el medio ambiente y la salud humana cuando se descarga sin el tratamiento adecuado ${ }^{2}$. Esta industria consume grandes volúmenes de agua, aproximadamente 100 litros de agua por kilogramo de tela teñida ${ }^{3,4}$.

Una de las alternativas para la mitigación de los efluentes de la industria textil de lana es la aplicación de tratamientos efectivos de bajo coste. El tratamiento fisicoquímico de 
coagulación-floculación es un método alternativo frente a otros tratamientos que generan mayores gastos a las empresas, elevando los costos de producción en el tratamiento de sus aguas residuales ${ }^{5,6}$.

Enríquez ${ }^{7}$, planteó una tecnología combinada de tratamiento anaerobio usando un reactor anaerobio de flujo ascendente y un reactor insuflado con aire, para tratar aguas procedentes del lavado de lana de oveja de la industria textil. Luego de combinar los dos tratamientos se obtuvieron porcentajes de remoción de $87,05 \%$ demanda bioquímica de oxígeno DBO5; 85,04 \% demanda química de oxígeno DQO; 88,52 \% sólidos totales suspendidos SST y 89,75 $\%$ aceites y grasas, cumpliendo con la normativa de la descarga de efluentes industriales. Se estudió el tratamiento de aguas residuales textiles a escala de laboratorio con los procesos: coagulación-floculación, biológico anaerobio y biológico aerobio, teniendo como resultado una remoción de más del $50 \%$ de los parámetros de DQO total, DQO soluble, DBO5 y color. Se observó que el coagulante Roquat-ATR produce una mejora en la eliminación de DQO, color y turbidez frente al cloruro ferroso ${ }^{8}$.

En la ciudad de Arequipa la mayoría de empresas de la industria textil no realizan el tratamiento adecuado a sus efluentes, motivo por el cual nuestro objetivo para la presente investigación fue evaluar el tratamiento fisicoquímico de los efluentes de lavado de lana provenientes de una industria textil de la ciudad de Arequipa con la finalidad de cumplir con los valores máximos admisibles (VMA) utilizando coagulante tricloruro férrico al $40 \%$ y floculante polímero MT-FLOC 4299 al 0,1 \%.

\section{PARTE EXPERIMENTAL}

\section{Muestras de efluente textil}

Se tomaron muestras compuestas de la matriz del agua residual de la industria textil ubicada en el parque industrial de Arequipa del proceso de lavado de lana por un tiempo de 24 horas. Se preservaron para análisis posterior de acuerdo al protocolo de monitoreo de efluentes aprobado por la $\mathrm{RM} \mathrm{N}^{\circ} 026-2000-\mathrm{ITINCI} / \mathrm{DM}^{9}$.

Se determinaron los parámetros físico-químicos DQO, DBO5, TSS, aceites y grasas ${ }^{10,11,12,13}$ establecidos en el anexo 1 que recomienda la norma vigente $\mathrm{DS}^{\circ}{ }^{\circ} 010-2019-$ VIVIENDA $^{14}$ en el efluente.

\section{Proceso de coagulación-floculación}

Antes de empezar las pruebas experimentales con el efluente se procede a homogenizar la muestra con un agitador tipo mariposa por 10 minutos, según se indica en la figura 1. 


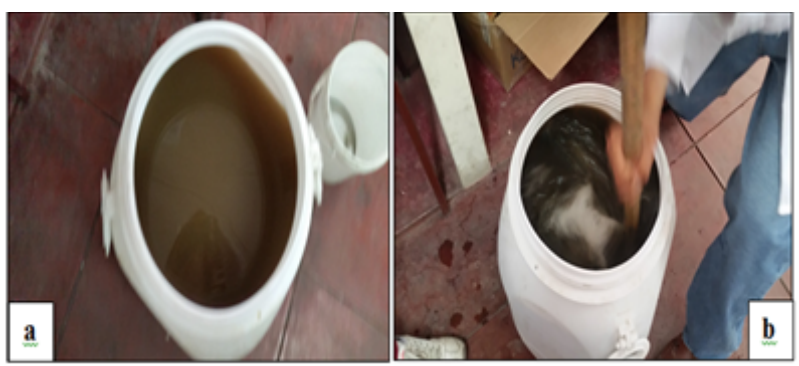

Figura 1. (a) Muestra de efluente textil. (b) Homogenización de la muestra Fuente: Elaboración propia.

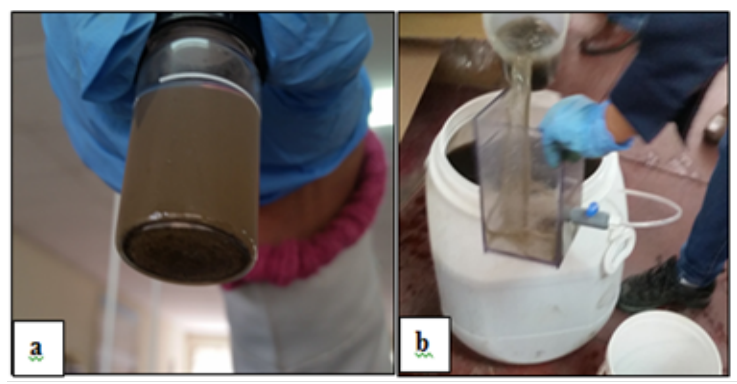

Figura 2. (a) Detección de inhibidores. (b) Carga del efluente en equipo de prueba de jarras. Fuente: Elaboración propia.

En la figura 2 se muestra la detección de aceites y grasas como posibles inhibidores de la coagulación - floculación, y el llenado del efluente textil en el equipo de prueba de jarras.

La muestra textil se caracterizó antes de realizar la prueba de jarras: temperatura $22,6^{\circ} \mathrm{C}, \mathrm{pH}$ promedio 7,65 y turbidez en el rango de 200 a 800 NTU.

Para determinar el rango de dosis óptima del coagulante y floculante se realizaron pruebas preliminares durante dos semanas con tres repeticiones al día, contabilizando un total de 30 repeticiones en la prueba de jarras modelo Phipps \& Bird digital programable.

Se ajustó la velocidad de las paletas de la prueba de jarras a $50 \mathrm{rpm}$ para uniformizar la muestra. Después se dosificó el coagulante tricloruro férrico al $40 \%$ a diferentes concentraciones con un tiempo de mezcla rápida de un minuto a $200 \mathrm{rpm}$ según se indica en la tabla 1. 
Tabla 1. Dosificación preliminar de coagulante, $\mathrm{FeCl}_{3}$ a $200 \mathrm{rpm}$.

\begin{tabular}{ccccccc}
\hline Concentración & \multicolumn{6}{c}{ Tratamientos } \\
\cline { 2 - 7 } $\mathrm{FeCl}_{3} 40 \%(\mathrm{mg} / \mathrm{L})$ & 1 & 2 & 3 & 4 & 5 & 6 \\
\hline
\end{tabular}

Fuente: Elaboración propia.

Para estos seis tratamientos preliminares se redujo la velocidad de agitación de las paletas a $40 \mathrm{rpm}$ por 15 minutos observando el tamaño de formación del flóculo.

En la figura 3. (a) y (b) se observan los dosificadores y flóculos mediante el índice de Willcomb, dejando sedimentar por 10 minutos.
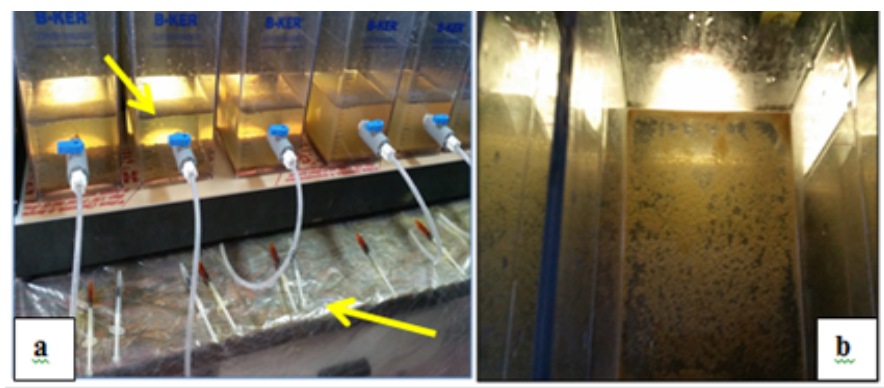

Figura 3. (a) Tamaño de flóculo y dosificadores. (b) Formación del coágulo. Fuente: Elaboración propia.

Para la determinación de la turbidez residual del agua decantada, se tomó la muestra directamente de los grifos de cada jarra y se expresaron en unidades nefelométricas de turbidez $(\mathrm{NTU})^{15,16}$. Se realizó la caracterización de los parámetros fisicoquímicos del agua tratada $^{17}$, figura 4 (a) y (b).

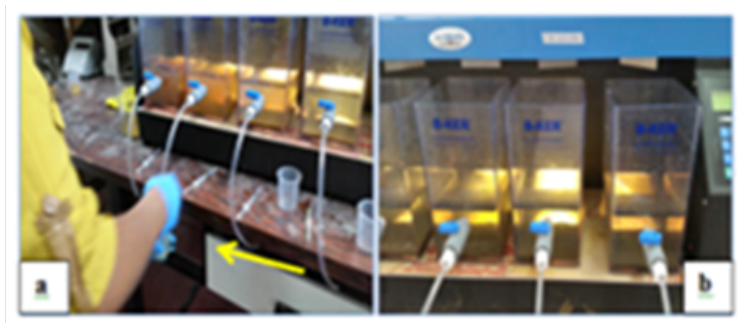

Figura 4. (a) Toma de muestra para determinación de turbidez y parámetros fisicoquímicos.

(b) Agua tratada. Fuente: Elaboración propia. 
Después de determinar la dosis óptima del coagulante ${ }^{18}$, bajo las condiciones operacionales (número de líneas y tipo de compósito) se decidió extender el rango de coagulante y las concentraciones de floculante como se muestra en la tabla 2 manteniendo el sistema a 200 $\mathrm{rpm} / \mathrm{min}$ y $40 \mathrm{rpm} / 15 \mathrm{~min}$. En la tabla 2 se observan las concentraciones de coagulante y floculante propuestas.

Tabla 2. Dosis del coagulante: $\mathrm{FeCl} 3$ al $40 \%$ y floculante: MT-FLOC.

\begin{tabular}{lccc}
\hline Concentración & Tratamientos \\
& 1 & 2 & 3 \\
\hline
\end{tabular}

Coagulante: $\mathrm{FeCl}_{3} 40 \%$

$\begin{array}{cccc}(\mathrm{mg} / \mathrm{L})(\text { Concentración) } & 200 & 220 & 240 \\ (\mathrm{~mL})(\text { Volumen }) & 0,5 & 0,55 & 0,6\end{array}$

Floculante $0,1 \%(\mathrm{mg} / \mathrm{L})$

$\begin{array}{llll}\text { (Concentración) } & 0,4 & 0,8 & 1,2 \\ (\mathrm{~mL}) \text { (Volumen) } & 0,2 & 0,4 & 0,6\end{array}$

Fuente: Elaboración propia.

En la figura 5 se muestra los tratamientos por duplicado para dosis de coagulante y floculante (mL) (volumen).

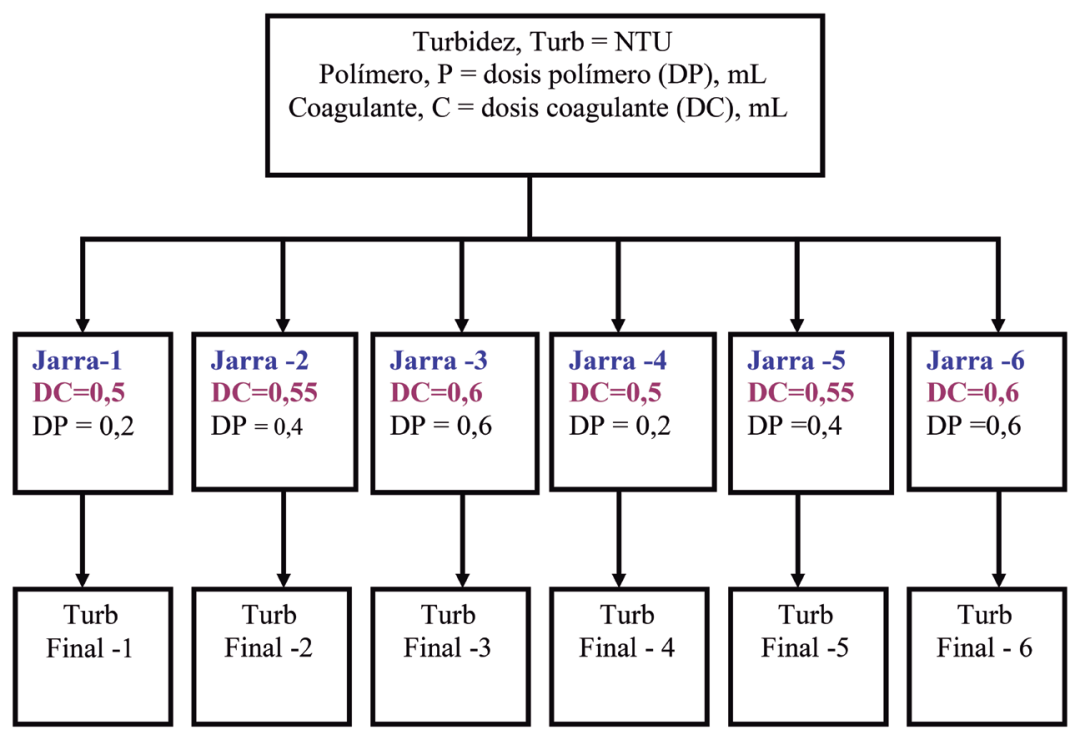

Figura 5. (a) Detección de inhibidores. (b) Carga del efluente en equipo de prueba de jarras. Fuente: Elaboración propia. 
En la figura 5 se observa un esquema de los tratamientos por duplicado, para las dosis de coagulante y floculante. Para cada dosis de floculante se realizaron cuatro repeticiones trabajando con tres dosis de coagulante óptimo en rango amplio.

Se trabajó en bloques y se observó que la dosis de rango amplio de coagulante manifestaba un mismo comportamiento, por lo que se tomó $0,5 \mathrm{~mL}$ como dosis óptima de coagulante. Con la dosis óptima se decidió ensayar las tres dosis $(0,4 ; 0,8$ y 1,2 mg/L) del floculante MTFLOC 4299 como se indicó en la tabla 2.

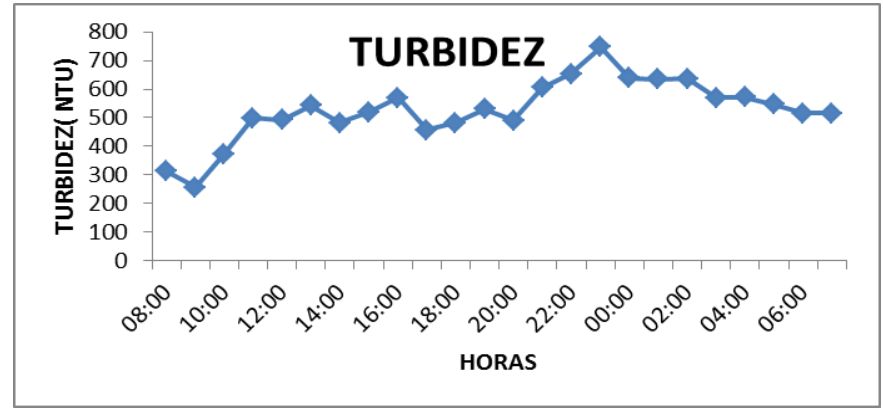

Figura 6. Turbidez durante 24 horas. Fuente: Elaboración propia.

La figura 6 muestra el rango de turbidez inicial del efluente de lavado de lana medido durante un periodo de 24 horas.

\section{RESULTADOS Y DISCUSIÓN}

\section{Determinación de dosis óptima del coagulante}

La muestra de agua residual de lavado de lana mostró valores de turbidez en el rango de 200 a 800 NTU. Se evaluó el efecto que ejerce el coagulante durante dos semanas. Previo al tratamiento estadístico se realizó un análisis exploratorio con datos de turbidez menor, igual o mayor a 600 NTU. En la figura 7 se observa que el rango óptimo de la dosis del coagulante se encuentra entre los valores de $0,5 \mathrm{~mL}$ y $0,6 \mathrm{~mL}$. 


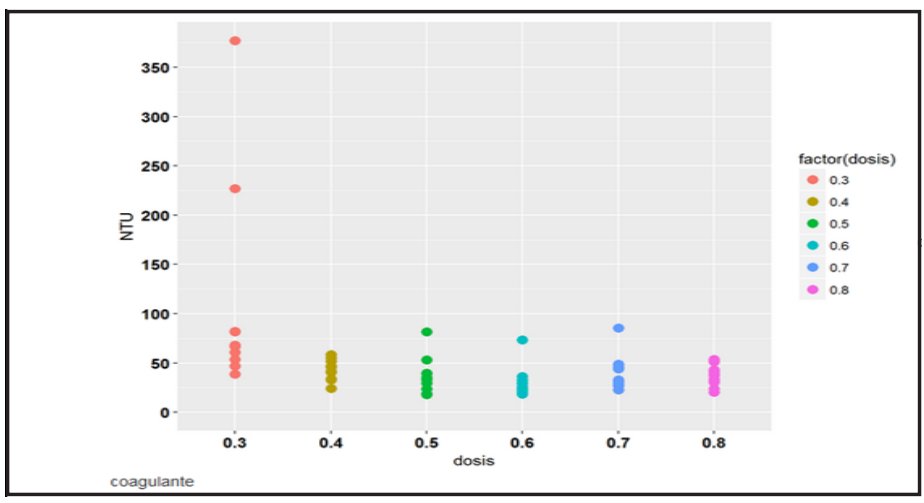

Figura 7. Turbidez final en cada nivel de coagulante.

Fuente: Elaboración propia.

Las comparaciones múltiples indican que existe un efecto similar en la reducción del nivel de turbidez cuando la dosis de coagulante es mayor que $0,5 \mathrm{~mL}(200 \mathrm{mg} / \mathrm{L})$.

\section{Dosis óptimas floculante y coagulante}

El tratamiento estadístico fue en bloques (figura 8), ya que se extendió la dosis óptima de coagulante a $0,5-0,55$ y $0,60 \mathrm{~mL}$ equivalente a $200 ; 220$ y $240 \mathrm{mg} / \mathrm{L}$ con las dosis de floculante 0,$2 ; 0,4$ y $0,6 \mathrm{~mL}$ equivalente a 0,$4 ; 0,8$ y $1,2 \mathrm{mg} / \mathrm{L}$ (tabla 2 ).

Nivel de confianza al $90 \%$

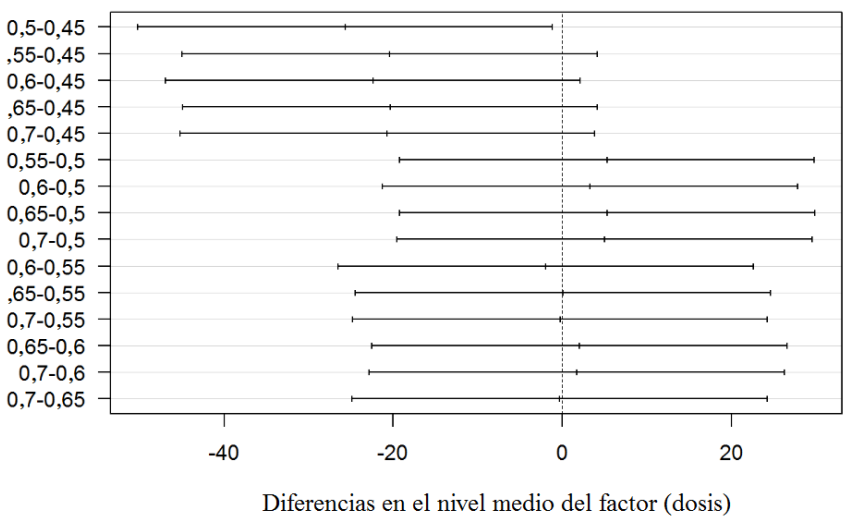

Figura 8. Prueba de Tukey.

Fuente: Elaboración propia. 
En la figura 8 se muestra que la mayoría de comparaciones múltiples tienen el mismo comportamiento para el nivel de turbidez, excepto para la comparación de 0,5-0,45 según la prueba de Tukey.

Según la prueba estadística, las dosis adecuadas fueron de $200 \mathrm{mg} / \mathrm{L}$ de coagulante y 0,8 $\mathrm{mg} / \mathrm{L}$ de floculante, obteniendo la mayor remoción para las aguas industriales de la industria textil.

La tabla 3 muestra parámetros medidos tanto para el efluente y para el agua tratada. Observamos que la demanda bioquímica de oxígeno y la demanda química de oxígeno, aceites y grasas manifiestan un alto grado de disminución, logrando un tratamiento eficiente con el coagulante y floculante evaluado18,19. Se formó una cantidad mínima de espuma que no interrumpió el tratamiento. La medición de los sólidos suspendidos totales TSS nos indicó la eficiencia de la remoción y la medición de aceites y grasas ${ }^{17}$. Se determinó la presencia de inhibidores que fueron también removidos con el tratamiento realizado.

Tabla 3. Dosificación preliminar de coagulante, $\mathrm{FeCl}_{3}$ a $200 \mathrm{rpm}$.

\begin{tabular}{|c|c|c|c|c|}
\hline Parámetros & Unidad & $\begin{array}{c}\text { AGUA } \\
\text { RESIDUAL } \\
\text { Resultado }\end{array}$ & $\begin{array}{c}\text { AGUA } \\
\text { TRATADA } \\
\text { Resultado }\end{array}$ & $\begin{array}{c}\text { VMA } \\
\text { Normativa } \\
\text { Peruana }\end{array}$ \\
\hline \multicolumn{5}{|l|}{ Parámetros Físico Químicos } \\
\hline Temperatura & ${ }^{\circ} \mathrm{C}$ & 22,6 & 22,4 & - \\
\hline $\mathrm{pH}$ & $\mathrm{pH}$ & 7,65 & 6,90 & - \\
\hline Turbidez & NTU & 526,96 & 57,97 & \\
\hline Sólidos Totales Suspendidos & $\mathrm{mg} / \mathrm{L}$ & 608 & $<2,5$ & 500 \\
\hline Sólidos Sedimentables & $\mathrm{mL} / \mathrm{L} / \mathrm{h}$ & 3,0 & $<0,1$ & - \\
\hline \multicolumn{5}{|l|}{ Parámetros Inorgánicos No } \\
\hline \multicolumn{5}{|l|}{ Metálicos } \\
\hline Cianuro Total & $\mathrm{mg} / \mathrm{L}$ & 0,0087 & 0,0021 & 1 \\
\hline Nitrógeno Amoniacal & $\mathrm{mg} / \mathrm{L}$ & 10,9 & 9,63 & \\
\hline Sulfuros & $\mathrm{mg} / \mathrm{L}$ & 0,0136 & 0,0107 & \\
\hline \multicolumn{5}{|l|}{ Aniones por Cromatografía } \\
\hline \multicolumn{5}{|l|}{ Iónica } \\
\hline Sulfatos, $\mathrm{SO}_{4}{ }^{2-}$ & $\mathrm{mg} / \mathrm{L}$ & 295 & 278 & \\
\hline \multicolumn{5}{|l|}{ Parámetros Orgánicos } \\
\hline Aceites y Grasas & $\mathrm{mg} / \mathrm{L}$ & 51,9 & 7,8 & 100 \\
\hline $\begin{array}{c}\text { Demanda Bioquímica de } \\
\text { Oxígeno }\end{array}$ & $\mathrm{mg} / \mathrm{L}$ & 619 & 108 & 500 \\
\hline Demanda Química de Oxígeno & $\mathrm{mg} / \mathrm{L}$ & 1407,9 & 290,6 & 1000 \\
\hline \multicolumn{5}{|l|}{ Metales } \\
\hline Cromo Hexavalente & $\mathrm{mg} / \mathrm{L}$ & $<0,001$ & $<0,001$ & 0,5 \\
\hline
\end{tabular}

Fuente: Elaboración propia. 


\section{CONCLUSIONES}

La dosis óptima del coagulante $\mathrm{FeCl} 3$ al $40 \%$ fue de $200 \mathrm{mg} / \mathrm{L}$ y del floculante MT-FLOC de $0,8 \mathrm{mg} / \mathrm{L}$, que fue determinante en la formación de flóculos más pesados, dando lugar a un incremento de la velocidad de sedimentación, obteniéndose una remoción del 84,97 \% de grasas, 82,55 \% de DBO5, el 79,36 \% de DQO y el 99,60 \% de sólidos totales suspendidos. Es factible aplicar el tratamiento de coagulación-floculación en agua residuales de lavado de lana para disminuir los parámetros antes mencionados cumpliendo con la normativa, $\mathrm{DS}^{\circ}$ 010-2019- VIVIENDA.

\section{AGRADECIMIENTO}

A la Universidad Nacional de San Agustín de Arequipa que financió el proyecto de investigación: "Tratamientos fisicoquímicos con métodos convencionales y avanzados de efluentes de la industria textil para el cumplimiento de los valores máximos admisibles (VMA) y su disposición al alcantarillado público", con el número de contrato IBA-00192016.

\section{REFERENCIAS BIBLIOGRÁFICAS}

1. Guillén M.O. Tratamiento fisicoquímico de aguas de lavado de lana. Universidad Nacional de San Agustín de Arequipa. [Tesis para optar el grado de Magister en Química]. Arequipa: Universidad Nacional de San Agustín; 2007.

2. Araújo CMB, Oliveira do Nascimento GF, Bezerra da Costa GR, Baptisttella AMS, Fraga TJM, de Assis Filho RB, et al. Real textile wastewater treatment using nano graphene-based materials: Optimum $\mathrm{pH}$, dosage, and kinetics for colour and turbidity. Can J Chem Eng. 2020; 98(6): 1429-1440.

3. Crites T. Tratamiento de aguas residuales en pequeñas poblaciones. Santa Fé de Bogotá, Colombia: McGraw-Hill Interamericana S.A.; 2000.

4. Seoánez C M. Manual de las aguas residuales industriales. Primera Edición. Madrid, España: Editorial McGraw-Hill; 2012.

5. Pérez V. Optimización del tratamiento primario avanzado con diagramas de coagulación - floculación del afluente de la Planta de Tratamiento de Aguas Residuales de Canaveralejo. Cali: Escuela Universidad del Valle; 2005.

6. Rivas - Romero, Sorangel. Tratamiento por coagulación - floculación a efluente de la Empresa del Níquel Ernesto Che Guevara. RTQ. 2017; 37(2): 173-183.

7. Enríquez FP. Tratamiento de aguas residuales del lavado de lana de oveja en la industria textil mediante un sistema combinado de reactores anaerobio/aerobio. [Tesis para optar el Título Profesional de Ingeniero Biotecnólogo]. Arequipa: Universidad Católica de Santa María; 2018. 
8. Perea MB. Estudio de tratabilidad de agua residual de industria textil a escala de laboratorio. [Tesis para optar el Título Ingeniero Técnico Industrial Especialidad en Química Industrial]. Santander: Universidad de Cantabria. Santander; 2012.

9. RM No 026-2000-ITINCI/DM. Protocolos de monitoreo de efluentes líquidos y emisiones atmosféricas. [Internet]. Lima, Diario oficial El Peruano; 2000. [Consultado 7 ago 2018]. Disponible en: https://www.legislacionambientalspda.org.pe/images/stories/ normas/Pioner\%202/IV.\%204.\%20Industrial/4.\%20Resoluciones\%20ministeriales/ Resolucion\%20ministerial\%20026-2000-ITINCI-DM.doc

10. 5220 Chemical oxygen demand (COD). Standard Methods For the Examination of Water and Wastewater. doi: 10.2105/SMWW.2882.103

11. 5210 biochemical oxygen demand (BOD). Standard Methods For the Examination of Water and Wastewater. doi: 10.2105/SMWW.2882.102

12. 2540 Solids. Standard Methods For the Examination of Water and Wastewater. doi: 10.2105/SMWW.2882.030

13. Method 1664, Revision B: n-HexaneExtractable Material (HEM; Oil andGrease) and Silica Gel Treatedn-Hexane Extractable Material (SGT-HEM; Non-polar Material) byExtraction and Gravimetry. [Internet]. [consultado 18 abril 2019]. Disponible en: https://www.epa.gov/sites/production/files/2015-08/documents/method_1664b_2010. pdf

14. DS N ${ }^{\circ}$ 010-2019-VIVIENDA. Reglamento de Valores Máximos Admisibles (VMA) para las descargas de aguas residuales no domésticas en el sistema de alcantarillado sanitario. Diario oficial El Peruano [Internet]. 2019. [Consultado 5 may 2019]. Disponible en: https://busquedas.elperuano.pe/download/url/decreto-supremo-que-aprueba-elreglamento-de-valores-maximos-decreto-supremo-n-010-2019-vivienda-1748339-3

15. MINAM. Estándares nacionales para la calidad ambiental del agua. DS. 002-2008-MINAM.[Internet].[Consultado 12 mayo 2019]. Disponible en: http://www. minam.gob.pe/calidadambiental/wp-content/uploads/sites/22/2013/10/ds_002_2008_ eca_agua.pdf

16. Sawyer CN, McCarthy PL, Parkin GF. Cuarta edición. Química para Ingeniería Ambiental. Bogotá: McGraw-Hill; 2011.

17. APHA-AWWA-WPCF. Métodos normalizados para el análisis de aguas potables y residuales. México: Ediciones Díaz de Santos S.A.; 1992. ISBN: 9788479780319

18. Degremont. Water Treatment Handbook. [Internet]. 2016. [consultado 10 abril 2019] Disponible en: https://www.iagua.es/noticias/degremont/16/09/01/ya-esta-disponiblemanual-tecnico-degremontr-suez-version-digital.

19. Labanda J, Llorens J. Wool scouring waste treatment by a combination of coagulationflocculation process and membrane separation technology. Chem Eng Process Intensif. 2008; 47(7):1061-8. 